\title{
Mobility Estimation Using an Extended Kalman Filter for Unmanned Ground Vehicle Networks
}

\author{
Preetha Thulasiraman*, Grace A. Clark ${ }^{\dagger}$, Timothy M. Beach* \\ * Department of Electrical and Computer Engineering \\ Naval Postgraduate School, Monterey, CA, USA \\ pthulas1@nps.edu, tmbeach@nps.edu \\ $\dagger$ Grace A. Clark, Ph.D., Consulting \\ (formerly with the Lawrence Livermore National Lab, July 1974-Oct. 2013) \\ clarkga1@comcast.net
}

\begin{abstract}
An ad hoc unmanned ground vehicle (UGV) network operates as an intermittently connected mobile delay tolerant network (DTN). In this paper, we develop a mobility estimation algorithm that can be coupled with a cooperative communication routing algorithm to provide a basis for real time path planning in UGV-DTNs. A Gauss-Markov state space model is used for the node dynamics. The nonlinear measurement signals are constant-power RSSI (Received Signal Strength Indicator) signals transmitted from fixed-position base stations. An extended Kalman filter (EKF) is derived for estimating the position, velocity and acceleration of a UGV node in a twodimensional spatial grid environment. We use Matlab to simulate a single mobile node traveling along a trajectory that includes abrupt maneuvers. Estimation performance is measured using zero-mean whiteness tests on the innovations sequences, root mean square error (RSME) of the state estimates, weighted sum squared residuals (WSSRs), and the posterior Cramer-Rao lower bound (PCRLB). Under these performance indices, we demonstrate that the mobility estimation algorithm performs effectively.
\end{abstract}

\section{INTRODUCTION}

In recent years, there has been increasing interest in the design and deployment of autonomous unmanned vehicles. In particular, one of the areas that has gained attention is the deployment of unmanned ground vehicles (UGVs). The Navy, Marine Corps and Army have invested monetary resources to the development of UGVs because of their potential to operate in a wide variety of situations [1]. With the introduction of unmanned vehicles, the traditional concept of warfare has shifted to a network centric view of military systems. This involves the integration of communication networking and information sharing into tactical military operations. This shift towards network centric warfare has led to the need for robust and reliable communications among groups of UGVs.

A UGV network operates as an intermittently connected mobile ad hoc network, otherwise known as a delay tolerant network (DTN) [2]. The ability to implement effective communication protocols among UGVs depends on the strategy of understanding the environment in which they are deployed. This is known as situational awareness and includes a real-time understanding of the terrain and activities of other UGVs in the same command. A primary factor in obtaining information for situational awareness is the dynamic mobility of each individual UGV. Obtaining knowledge about the mobility of the UGVs requires estimation of the position, velocity and acceleration of the UGV node at a given time and is an integral part of the path planning strategy.

In this regard, the overall UGV-DTN system design requires solution of the following two component problems:

- Mobility Estimation: We must develop a mobility estimation algorithm that will achieve realistic estimates of the positions of the individual UGV nodes within the DTN.

- Path Planning: We must develop a path planning strategy using the mobility estimation results as inputs to achieve cooperation among individual UGV nodes for routing.

This paper focuses on the mobility estimation problem. The development of the path planning algorithm and the integration of the two components is left for future research.

\section{A. Contributions}

This paper studies the creation of a new mobility estimation approach that exploits a general two-dimensional spatial grid setting, a Gauss-Markov state space dynamic model, and a first order semi-Markov model for the command action. To the best of our knowledge, this is the first work that integrates the use of signal processing and control techniques for mobility estimation in a wireless network of UGV nodes. Our contribution in this paper can be summarized as follows: We develop a mobility estimation algorithm in an ad hoc setting for both linear and non-linear models, based on an extended Kalman Filter (EKF). Specifically, Gauss-Markov and semi-Markov type signal models along with the EKF estimation algorithm are exploited for use in the UGV-DTN mobility estimation algorithm. The discrete-time nonlinear Gauss Markov model and discrete time EKF algorithm are derived for the UGVDTN and simulated using Matlab. Performance measures are analyzed for the EKF mobility estimation algorithm.

The remainder of the paper is organized as follows. In Section II, the related work is discussed. The models used for the mobile node, and the Jacobian matrix are given in Section III. The EKF mobility estimation algorithm is formulated and discussed in Section IV. The performance evaluation is given in Section V. We conclude the paper in Section VI. 


\section{RELATED WORK}

The mobility estimation literature for an ad hoc network focuses on determining node coordinates using measured signals from known base stations (BSs), the GPS, etc.

This type of setting is generally most appropriate for mobile ad hoc networks based on a DTN architecture, particularly in military applications. The measurements in this setting usually involve RSSI signals from known base stations [3], [4]. The signal model is usually some form of Gauss-Markov model. These can include auto-regressive (AR) moving average (ARMA) models and their many variants [5], [6], [7]. These models are sometimes augmented with a semiMarkov model to represent uncertain accelerations, etc. [8], [9].The performance indices generally include mean squared error (MSE), the Cramer-Rao lower bound (CRLB), and the posterior CRLB (PCLRB) [9], [6]. The estimation algorithms usually consist of a Kalman Filter (KF), EKF, Unscented KF or particle filter $(\mathrm{PF})$, also called a sequential Monte Carlo (SMC) filter, depending on whether or not the system model is linear or nonlinear and the uncertainties (noise processes) are Gaussian or Non-Gaussian. Some published algorithms assume that the model parameters are known a priori [14].

Zaidi et al. [4] study ad hoc networks with intermittent connectivity. The algorithm for mobility tracking developed in [4] uses RSSI measurements from neighboring nodes modeled as a linear system driven by a discrete semi-Markov process in combination with an efficient averaging filter and an EKF. The proposed algorithm allows robust mobility tracking in ad hoc networks using RSSI measurements. Root MSE (RMSE) is used as the performance measure. The algorithm is able to follow mobile trajectories accurately over a wide range of parameter values. The proposed mobility tracking algorithm can be applied in a variety of scenarios, such as adaptive clustering, routing, and mobility management in ad hoc networks [4].

Yang et al. [10] consider a SMC method for joint mobility tracking and cellular handoff in wireless communication networks. The mobility tracking is based on the measurement of RSSI signals from known base stations. The system dynamics are described by a nonlinear state space model. The mobility tracking includes estimation of the position and velocity of the mobile node. The EKF is identified as the main technique for solving online estimation in a nonlinear dynamic system. Using the SMC framework, Yang et al. jointly solve the problem of online estimation and online prediction of RSSI at some future time instance. The SMC was compared with the modified EKF and was shown to improve tracking accuracy and minimize the tradeoff between quality of service (QoS) and resource utilization [18]. However, the SMC-based approach comes with a significantly high computational cost.

Mihaylova et al. [3] also consider a SMC technique for mobility tracking in wireless communication networks by means of RSSI. The technique allows for estimation of mobile position, velocity and acceleration. $\mathrm{A}$ PF and RBPF are proposed and analyzed over real and simulated data. A com- parison with an EKF is performed with respect to accuracy and computational complexity in scenarios with abrupt maneuvers. Advantages of the RBPF compared with the PF are decreased computational complexity exhibiting similar accuracy and smaller peak-dynamic errors during abrupt maneuvers, which is important for practical purposes [13]. In this paper, we adapt the state space mobility model from [3].

\section{Mobility Estimation Models}

The mobility estimation algorithm design requires several key problem specifications, or attributes. The key attributes are as follows: (1) the operational mission setting and physical constraints, (2) the set of available sensor measurements or observations, (3) an appropriate physics model, (4) an appropriate performance index or set of performance indices, and (5) an appropriate estimation/tracking algorithm or set of algorithms. We describe and compose an algorithm in terms of these five key attributes.

\section{A. UGV-DTN System Model}

The network environment considered in this paper is shown in Fig. 1. Spatially distributed UGV cluster islands are assumed to be connected via an unmanned aerial vehicle (UAV) which can act as a relay node to carry information among groups of UGV cluster islands. No direct communication exists among nodes of different cluster islands. UGVs within a cluster island communicate cooperatively to forward messages from source to destination within one cluster island. Geo-location using the Global Positioning System (GPS) is assumed to be available on an UAV and on a sub-set of UGV nodes, which carry GPS in addition to their built-in received signal strength indicator (RSSI) sensors. This paper explores mobility estimation within one individual UGV cluster island. The UAV and all of the UGV nodes contain sensors that produce measurements as indicated in Fig. 2. The mobile nodes and the UAV pass measurement signals/data to the routing and mobility predication algorithms. The purpose of the mobility estimation algorithm is to produce estimates of position, velocity and acceleration versus time for a particular node or nodes in the cluster island. To further breakdown the overall problem, the measurements obtained from one UGV node will be used to construct the measurement vector for the mobility estimation algorithm as shown in Fig. 3.

\section{B. Mobility Estimation Models}

For our analysis we chose to use a Gauss-Markov state space model [4]. The specific model chosen for the implementation of the mobility estimation of a UGV node in a UGV-DTN is a discrete-time variant of the Singer model originally proposed in [11]. Mihaylova et al. [3] has shown that the modified Springer model used by Yang and Wang [10] performs well, is simple, and allows efficient computation of performance indices. This is a Gauss-Markov type model modified to include a discrete semi-Markov type model for the input command function. The dynamic model for the mobile node is linear, but the measurement model is highly nonlinear. 


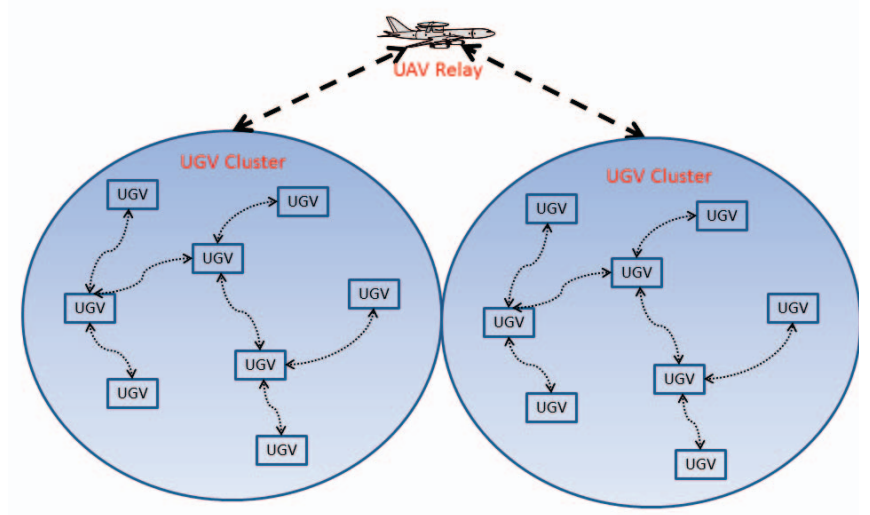

Fig. 1. A clustered UGV-DTN communicating wirelessly within each cluster and to and from a UAV

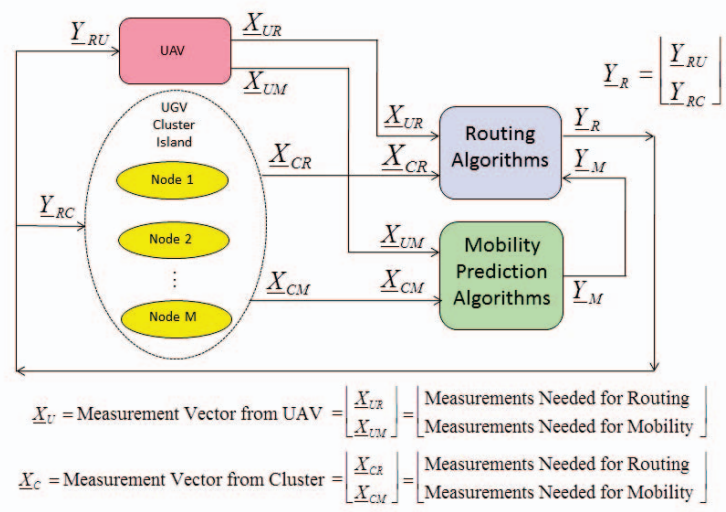

Fig. 2. Block diagram of the overall signal/data flow for a UGV-DTN

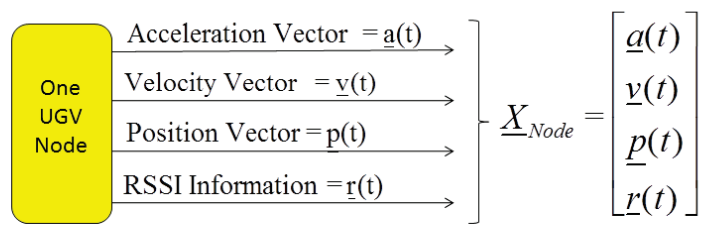

RSSI $=$ Received Signal Strength Indicator

$$
\Rightarrow \text { Power of the Signal Received at the Antenna }
$$

Fig. 3. Block diagram of the overall signal/data flow for one UGV-DTN node in a cluster
1) Model for the State of the Mobile Node: Let the twodimensional spatial coordinates be denoted by $(x, y)$. Let $k$ denote the discrete time index, and let $T$ denote the temporal sampling period. We let $\left(x_{k}, y_{k}\right)$ denote the position of the mobile node at discrete time $k$. We then denote the speed by $\left(\dot{x_{k}}, \dot{y_{k}}\right)$ and the acceleration by $\left(\ddot{x_{k}}, \ddot{y_{k}}\right)$. The state of the mobile node at discrete time $k$ is then denoted by $\underline{x}_{k}=\left[x_{k}, \dot{x_{k}}, \ddot{x_{k}}, y_{k}, \dot{y_{k}}, \ddot{y_{k}}\right]^{T}$ where the superscript $T$ denotes vector transpose. The linear state for the mobile node is given by:

$$
\underline{x}_{k}=A(T, \alpha) \underline{x}_{k-1}+B_{u}(T) \underline{u}_{k}+B_{w}(T) \underline{w}_{k}
$$

where $\underline{u}_{k}=\left[u_{x, k}, u_{y, k}\right]^{T}$ is the discrete time command process, or system input and $w_{k}=\left[w_{x, k}, w_{y, k}\right]$ is a white Gaussian noise sequence with zero mean and covariance matrix $Q=\sigma_{w}^{2} I$ where $I$ denotes the unit or identity matrix and $\sigma_{w}^{2}$ is the covariance of noise. The parameter $\alpha$ depends on the duration of a maneuver, and is the reciprocal of the maneuver time constant. Note that the matrix $A(T, \alpha)$ is a function only of the sampling period and the reciprocal of the maneuver constant, and the matrices $B_{u}(T)$ and $B_{w}(T)$ are functions only of the sampling period [3].

In the real world, a mobile node is likely to have both discontinuous motion and continuous motion. A mobile node is likely to have sudden and unexpected acceleration changes. Simultaneously, we must account for the fact that node acceleration is likely to be correlated over time, due to momentum. For these reasons, we model the mobile node as a dynamic system driven by a semi-Markov acceleration process $\underline{a}_{k}=$ $\underline{u}_{k}+\underline{r}_{k}$. This acceleration is the sum of a two-dimensional semi-Markov driving command $\underline{u}_{k}=\left[u_{x, k}, u_{y, k}\right]^{T}$ and a two-dimensional time-correlated random acceleration vector $\underline{r}_{k}=\left[r_{x, k}, r_{y, k}\right]^{T}$. Both $u_{x, k}$ and $u_{y, k}$ are independent semiMarkov processes acting in the $x$ and $y$ directions [4], [8], [11]. The model produces correlated random accelerations using a representative model of the autocorrelation function given by [8]

$$
R_{r r}(\tau)=E\left\{\underline{r}(t) \underline{r}^{T}(t+\tau)\right\}=\sigma_{m}^{2} e^{-\alpha|\tau|} I
$$

where $\alpha \geq 0, \sigma_{m}^{2}$ is the variance of the random acceleration in a single dimension, and $\alpha$ is the reciprocal of the random acceleration time constant. The final mobile node dynamic model is summarized in Eq. 3 .

$$
\begin{aligned}
\left(\begin{array}{c}
x_{k} \\
\dot{x}_{k} \\
\ddot{x}_{k} \\
y_{k} \\
\dot{y}_{k} \\
\ddot{y}_{k}
\end{array}\right)= & \left(\begin{array}{cccccc}
1 & T & \frac{T^{2}}{2} & 0 & 0 & 0 \\
0 & 1 & T & 0 & 0 & 0 \\
0 & 0 & \alpha & 0 & 0 & 0 \\
0 & 0 & 0 & 1 & T & \frac{T^{2}}{T} \\
0 & 0 & 0 & 0 & 1 & T \\
0 & 0 & 0 & 0 & 0 & \alpha
\end{array}\right)\left(\begin{array}{c}
x_{k-1} \\
\dot{x}_{k-1} \\
\ddot{x}_{k-1} \\
y_{k-1} \\
\dot{y}_{k-1} \\
\ddot{y}_{k-1}
\end{array}\right)+ \\
& \left(\begin{array}{cc}
\frac{T^{2}}{2} & 0 \\
T & 0 \\
0 & 0 \\
0 & \frac{T^{2}}{2} \\
0 & T \\
0 & 0
\end{array}\right)\left(\begin{array}{ll}
u_{x, k} \\
u_{y, k}
\end{array}\right)+\left(\begin{array}{cc}
\frac{T^{2}}{2} & 0 \\
T & 0 \\
1 & 0 \\
0 & \frac{T^{2}}{2} \\
0 & T \\
0 & 1
\end{array}\right)\left(\begin{array}{l}
w_{x, k} \\
w_{y, k}
\end{array}\right)
\end{aligned}
$$




$$
H=\left(\begin{array}{cccccc}
\frac{-10 \eta\left(x_{k, 1}-a_{1}\right)}{\ln (10)\left[\left(x_{k, 1}-a_{1}\right)^{2}+\left(x_{k, 4}-b_{1}\right)^{2}\right]} & 0 & 0 & \frac{-10 \eta\left(x_{k, 4}-b_{1}\right)}{\ln (10)\left[\left(x_{k, 1}-a_{1}\right)^{2}+\left(x_{k, 4}-b_{1}\right)^{2}\right]} & 0 & 0 \\
\frac{-10 \eta\left(x_{k, 1}-a_{2}\right)}{\ln (10)\left[\left(x_{k, 1}-a_{2}\right)^{2}+\left(x_{k, 4}-b_{2}\right)^{2}\right]} & 0 & 0 & \frac{-10 \eta\left(x_{k, 4}-b_{2}\right)}{\ln (10)\left[\left(x_{k, 1}-a_{2}\right)^{2}+\left(x_{k, 4}-b_{2}\right)^{2}\right]} & 0 & 0 \\
\frac{-10 \eta\left(x_{k, 1}-a_{3}\right)}{\ln (10)\left[\left(x_{k, 1}-a_{3}\right)^{2}+\left(x_{k, 4}-b_{3}\right)^{2}\right]} & 0 & 0 & \frac{-10 \eta\left(x_{k, 4}-b_{3}\right)}{\ln (10)\left[\left(x_{k, 1}-a_{3}\right)^{2}+\left(x_{k, 4}-b_{3}\right)^{2}\right]} & 0 & 0
\end{array}\right)
$$

\section{Measurement (Observation) Model}

The measurements consist of RSSI signals from knownlocation BSs. Locating a node in a two-dimensional spatial plane requires a minimum of three BSs. Increasing the number of BSs to seven will improve accuracy [3]. Let $M_{B S}$ denote the number of BSs. We are given measurements of the location $\left(a_{i, k}, b_{i, k}\right)$ of each of the BSs at discrete time $k$, where $i=i, \ldots, M_{B S}$. Let us denote the measurement model by a nonlinear vector equation of the form:

$$
\left.\underline{z}_{k}=\underline{h} \underline{x}_{k}\right]+\underline{v}_{k}
$$

where $\underline{z}_{k}$ denotes the measurement vector, $\underline{h}\left[\underline{x}_{k}\right]$ is a nonlinear function, and $\underline{v}_{k}$ is the measurement noise. The RSSI signal can be modeled as a sum of two terms: path loss $\left(\underline{h}\left[\underline{x}_{k}\right]\right)$ and shadow fading $\left(\underline{v}_{k}\right)$. The RSSI (measured in $\mathrm{dB}$ ) signal of a single BS is modeled by:

$$
\underline{z}_{k, i}=\underline{z}_{0, i}-10 \eta \log _{10}\left(d_{k, i}\left[\underline{x}_{k}\right]\right)+v_{k, i}
$$

where $\underline{z}_{0, i}$ is a constant characterizing the transmission power of the base station. It is a function of wavelength, antenna, height and gain of node $i$. The constant $\eta$ is called the slope index, and it takes on various values, depending on the characteristics of the physical environment (i.e., $\eta=2$ for highways, or 4 for microcells in a city). The distance $d_{k, i}\left[\underline{x}_{k}\right]$ is the distance between the mobile node and the base station $i$ at a discrete time $k$. The process $v_{k}=\left[v_{k, 1}, \ldots, v_{k, M_{B S}}\right]$ is the shadowing component.

\section{Derivation of Jacobian Matrix for EKF}

The EKF used for the estimation algorithm requires a Jacobian, or gradient matrix $H$ for approximate linearization of our non-linear measurement, where

$$
\left.H \triangleq \frac{\partial \underline{h}\left[\underline{x}_{k}\right]}{\partial \underline{x}_{k}}\right|_{\underline{x}_{k}=\underline{\hat{x}}_{k \mid k-1}}
$$

Due to space limitations, we do not include the derivation steps for the Jacobian. The final Jacobian matrix used in this work is given in Eq. 4 [12].

\section{Extended Kalman Filter Algorithm}

The EKF is a state space nonlinear state estimator that provides estimates of the state vector at each discrete time step $t$. It is the optimal least squares estimator for our model. The EKF is an extension of the KF, a wholly linear estimator, because it handles the nonlinear Gauss-Markov model. The following is notation used in this section: a "hat" above a symbol denotes an estimate (i.e., $\underline{\hat{x}}(t))$. A tilde above a symbol is used to denote an error (i.e., $\underline{\tilde{x}}(t \mid t-1)$ ). This is read as the error in the states at time step $t$, given data up to a time step $t-1$. A double tilde indicates an error covariance matrix [12], [13].

\section{A. Discrete Time Nonlinear Gauss-Markov Model}

Given state vector $\underline{x}(t)$, initial state vector $\underline{x}(0)$, system matrices $A(),. B_{u}($.$) , and B_{w}($.$) , system input vector \underline{u}(t)$ and process noise $\underline{w}(t)$, we can write the state propagation model as follows:

$$
\underline{x}(t)=A(T, \alpha) \underline{x}(t-1)+B_{u}(T) \underline{u}(t)+B_{w}(T) \underline{w}(t)
$$

Given the system output measurement vector $\underline{z}(t)=$ $\left[z_{1}(t), z_{2}(t), z_{3}(t)\right]^{T}$, nonlinear function $h($.$) , and measure-$ ment noise vector $\underline{v}(t)$, we can write the measurement propagation as follows:

$$
\underline{z}(t)=\underline{h}[\underline{x}(t)]+\underline{v}(t)
$$

Note that for our mobility problem, $\underline{w}(t)$ and $\underline{v}(t)$ are zeromean white Gaussian noise sequences.

\section{B. Discrete Time EKF Algorithm}

Given the nonlinear Gauss-Markov model for the mobile node, the derived EKF algorithm equations are summarized as follows:

1) Prediction: The state prediction is

$$
\underline{\hat{x}}(t \mid t-1)=A(T, \alpha) \underline{\hat{x}}(t-1 \mid t-1)+B_{u}(T) \underline{u}(t-1)
$$

and the state error covariance step is

$\tilde{\tilde{P}}(t \mid t-1)=A(T, \alpha) \tilde{\tilde{P}}(t-1 \mid t-1) A^{T}(T, \alpha)+\tilde{R}_{W}(t-1)$

Using Eqs. 10 and 11, we predict the next estimate of the state error covariance by propagating the state estimation error from the previous time step through the system matrix $A(T, \alpha)$ and adding process noise covariance.

2) Innovation: The innovations sequence is the error between the measured output and the estimated output

$$
\underline{e}(t)=\underline{z}(t)-\underline{\hat{z}}(t \mid t-1)
$$

and the innovation covariance is

$\tilde{R}_{e}(t)=\tilde{H}[\underline{\hat{x}}(t \mid t-1)] \underline{\tilde{P}}(t \mid t-1) \tilde{H}^{T}[\underline{\hat{x}}(t \mid t-1)]+\tilde{R}_{V}(t)$

The innovations vector is the difference between the current measurement and the last estimate of the measurement vector given data up to time $t-1$. The innovations represent new information available to the EKF since the last state update.Once the innovations vector is computed, we calculate the next value of the innovations covariance matrix using Eq. 13. 
3) Gain: The Kalman gain is

$$
\tilde{K}(t)=\underline{\tilde{\tilde{P}}}(t \mid t-1) \tilde{H}^{T}[\underline{\hat{x}}(t \mid t-1)] \tilde{R}_{e}^{-1}(t)
$$

The Kalman gain matrix provides the key factor we use in the next step to update the state estimate in a direction toward minimizing the MSE. A small value of the Kalman gain indicates that the filter places a large weight on the latest model predictions; whereas, a large gain indicates that the filter places a large weight on the latest measurements [4].

4) Correction: The state correction step is

$$
\underline{x}(t \mid t-1)=\underline{\hat{x}}(t \mid t-1)+\tilde{K}(t) \underline{e}(t)
$$

and the state error covariance estimator correction step is

$$
\underline{\tilde{\tilde{P}}}(t \mid t)=\{\tilde{I}-\tilde{K}(t) \tilde{H}[\underline{\hat{x}}(t \mid t-1)]\} \underline{\tilde{\tilde{P}}}(t \mid t-1)
$$

In this step, the filter updates (corrects) the last state estimate by adding to it the product of the Kalman gain matrix and the innovations vector. By doing so, it moves the state estimate in a direction that reduces the MSE. After many time steps, a properly tuned EKF will converge toward the minimum mean square error estimate of the states. In this correction step, the EKF also updates (corrects) the state estimation error covariance matrix as in Eq. 16.

5) Initial Conditions: The initial state vector estimate $\underline{\hat{x}}(0 \mid 0)$ and the initial state error covariance matrix are chosen and explored in Section V.

6) Jacobian Matrix: The Jacobian matrix given in Section III-D is used to linearize the nonlinear RSSI measurement equation given in Eq. 9.

\section{Performance Measures for the EKF}

The following section summarizes methods for evaluating the performance of the EKF and its application to the UGVDTN scenario.

- Zero-Mean Test: When the EKF is tuned, it provides the optimal mean squared error (MSE) estimate of the state vector. A necessary and sufficient condition for the EKF to be optimal is that the innovations sequence is zeromean and white [9]. We therefore use hypothesis testing to ascertain that the innovations have zero mean within a 95\% confidence interval, or "two-sigma bounds" [9], [12], [13].

- Whiteness Test: We use another hypothesis test to declare whether or not the innovations are white. The test statistic is the normalized sample auto-covariance function of an individual innovations sequence. Ideally, if the innovations are white, then the auto-covariance should be a Kronecker delta function. A 95\% confidence interval on the covariance values is computed. If fewer than 5\% of the N-1 samples in the covariance sequence that follow the sample at zero lag lie within the confidence interval, then the innovations are declared to be white [9], [12], [13].

- Root Mean Square Error (RMSE): The error in the states is given by $\underline{\tilde{x}}(t)=\underline{x}(t)-\underline{\hat{x}}(t \mid t-1)$. We compute and plot this error for each of the states as a measure of performance. The mean is estimated by averaging over 100 realizations of the stochastic process.

- Weighted Sum Squared Residuals (WSSR): The WSSR provides a method for whiteness testing over all of the innovations by aggregating innovations vector information into a single scalar test statistic. We use a hypothesis test to ascertain that the WSSR lies below a pre-determined threshold [9], [12], [13].

- Posterior Cramer-Rao Lower Bound (PCRLB): The PCRLB provides a lower bound on the state error covariance matrix $\tilde{\tilde{P}}(t \mid t-1)$. This tells us the best possible theoretical covariance that is attainable [3], [9], [12], [14].

\section{Simulation Experiments and Performance EVALUATION}

In this section, we present the simulation results conducted in Matlab to demonstrate and validate the EKF algorithm. We simulate a single mobile node traveling along a trajectory that includes abrupt maneuvers. We use a Gauss-Markov state space model for the node dynamics. Process noise is assumed to be zero. The measurements are constant power RSSI signals transmitted from fixed position base stations. We use the EKF derived in Section IV for state estimation, including node position coordinates in a two-dimensional spatial grid environment.

The simulation parameters used in Matlab are as follows: The discretization time step $(T)$ is 0.5 secs, correlation coefficient $(\alpha)$ is 0.6 , path loss index $(\eta)$ is 3 , base station transmission power $\left(z_{0, i}\right)$ is 90 , covariance $\left(\sigma_{w}^{2}\right)$ of the noise $w_{k}$ is $0.5^{2}\left[\mathrm{~m} / \mathrm{s}^{2}\right]^{2}$, covariance $\left(\sigma_{v}{ }^{2}\right)$ of the noise $v_{i, k}$ is $4^{2}[d b]^{2}$, the maximum speed $\left(V_{\max }\right)$ is $45 \mathrm{~m} / \mathrm{s}$, the transition probabilities $\left(p_{i, j}\right)$ are set to 0.8 , and the initial mode probabilities $\mu_{i, 0}$ are $1 / M, i=1, \ldots, M, M=5$. The parameters are chosen such that node behavior is realistic. In other words, abrupt maneuvers are included to test the estimator's ability to adapt to rapid trajectory changes.

The EKF initial state estimate is set to:

$$
\underline{\hat{x}}(0 \mid 0)=\left(\begin{array}{llllll}
3400 & 5 & 0 & 8700 & 8 & 0
\end{array}\right)^{T}
$$

The EKF initial covariance estimate is set to:

$$
\tilde{\tilde{P}}(0 \mid 0)=\left(\begin{array}{cccccc}
400^{2} & 0 & 0 & 0 & 0 & 0 \\
0 & 15^{2} & 0 & 0 & 0 & 0 \\
0 & 0 & 5^{2} & 0 & 0 & 0 \\
0 & 0 & 0 & 400^{2} & 0 & 0 \\
0 & 0 & 0 & 0 & 0 & 5^{2}
\end{array}\right)
$$

The initial state estimate was chosen based on the assumption that we have reasonable a priori knowledge of the initial states of the UGV node because we deploy the node ourselves. The initial covariance estimate is chosen based on the estimate we make on the standard deviations of the node states based on our knowledge of the operational environment and node capabilities. 


\section{A. Estimation of the States with the EKF}

The discrete-time input command process $u_{x, k}$ and $u_{y, k}$ is chosen to be a first order Markov chain that can take on discrete values $-3.5,0$ and 3.5 in units of $\mathrm{m} / \mathrm{s}^{2}$. The command input includes two turns, once at 150 secs and once at 200 secs. Please see [12] for further details.

A plot of the estimated track $\underline{\hat{x}}_{k \mid k-1}$ from the EKF overlayed on a plot of the actual trajectory to include base stations used for triangulation is shown in Fig. 4. After the initial track errors during the transient state, the estimation settles into a trajectory that tracks closely to the actual trajectory. A plot of the estimated root mean speed $\hat{\dot{x}}_{k}=\sqrt{\hat{x}_{2, k}^{2}+\hat{x}_{5, k}^{2}}$ overlayed on a plot of the actual root mean speed $\dot{x}_{k}=\sqrt{x^{2}{ }_{2, k}+x^{2}{ }_{5, k}}$ is illustrated in Fig. 5. The initial velocity errors settle after about 40 seconds of transient behavior and closely track the true velocity [12].

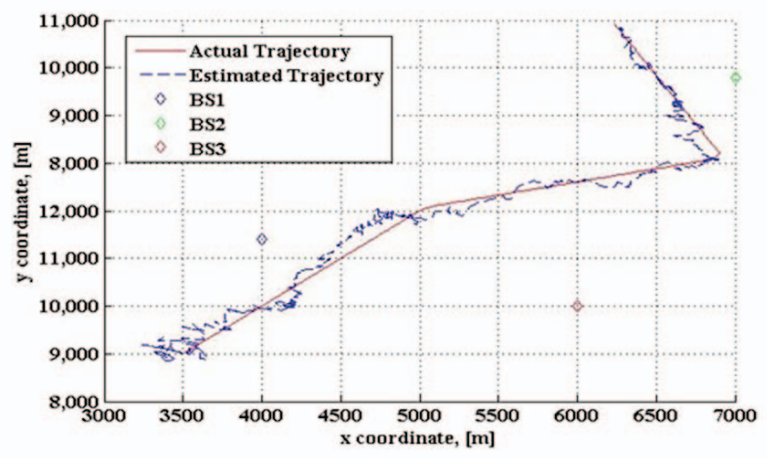

Fig. 4. Estimated track, simulated track, and locations of base stations transmitting RSSI signals used for triangulation of the UGV node
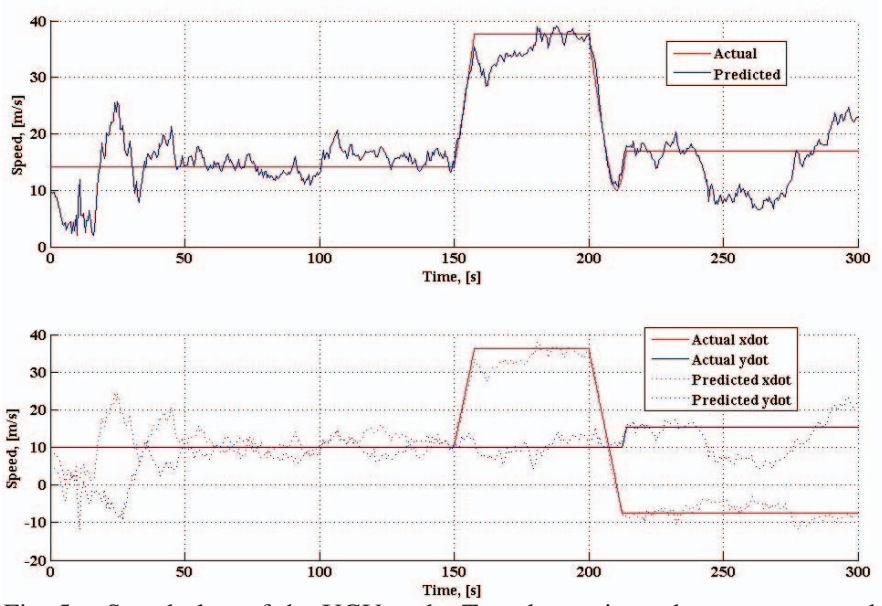

Fig. 5. Speed plots of the UGV node. Top plot: estimated root mean speed $\hat{\dot{x}}_{k}=\sqrt{\hat{x}_{2, k}^{2}+\hat{x}_{5, k}^{2}}$ and actual root mean speed $\dot{x}_{k}=\sqrt{x^{2}{ }_{2, k}+x^{2}{ }_{5, k}}$. Bottom plot: estimated $x$ and $y$ velocity, $\hat{x}_{2, k}$ and $\hat{x}_{5, k}$ and actual $x$ and $y$ velocity, $x_{2, k}$ and $x_{5, k}$ of the node

The error between the estimated and actual states $\underline{\tilde{x}}_{k}=$ $\underline{x}_{k}-\underline{\hat{x}}_{k \mid k-1}$ over time is presented in Fig. 6. The errors $\underline{\tilde{x}}_{k}$ are shown to be acceptable in that they are approximately zero mean and Gaussian in distribution. The errors are shown to be zero-mean and lie within the two sigma-bounds at three tenths of a percent deviation each.

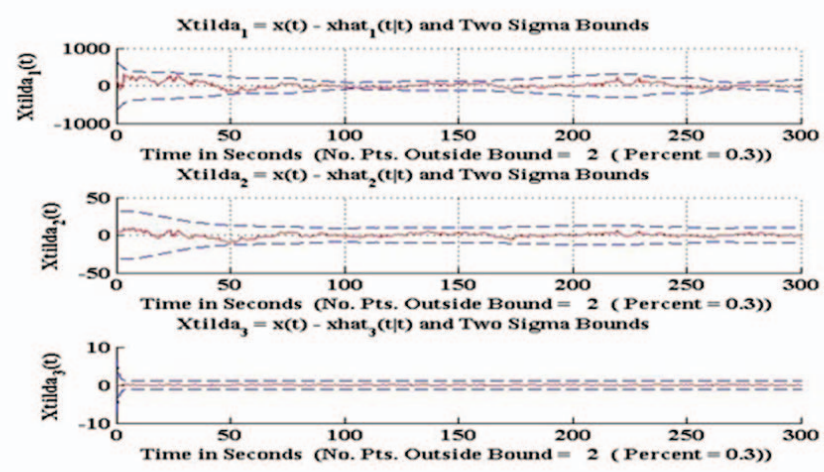

Fig. 6. Error between the estimated states and the actual states $\underline{x}_{k}=\underline{\hat{x}}_{k}-\underline{x}_{k}$ of the UGV nodes and their respective two sigma bounds plotted over time. The top plot corresponds to position, the middle plot corresponds to velocity, and the bottom plot corresponds to acceleration of the UGV node

\section{B. Performance and Tuning of the EKF}

The innovations were tested and shown to satisfy the zeromean and whiteness tests, as described earlier. Please see [12] for a complete description of the tests. Figures 7 and 8 depict a measure of the ability of the EKF to estimate the node trajectory. Figure 7 shows the position RMSE overlaid on the position PCRLB for the node. Figure 8 shows the velocity RMSE overlaid on the velocity PCRLB for the node. In both Figures 7 and 8, the means were estimated using 100 realizations of the stochastic processes. We see from Figure 7 that the position RMSE is within about 100 meters of the position PCRLB. In Figure 8, we see that the velocity RMSE is within about 5 meters of the velocity PCRLB. Given that the initial position standard deviation was $400 \mathrm{~m}$ and the initial velocity standard deviation was $15 \mathrm{~m} / \mathrm{sec}$, we see that the EKF estimates converged nicely toward the best possible theoretical values (the PCRLB).

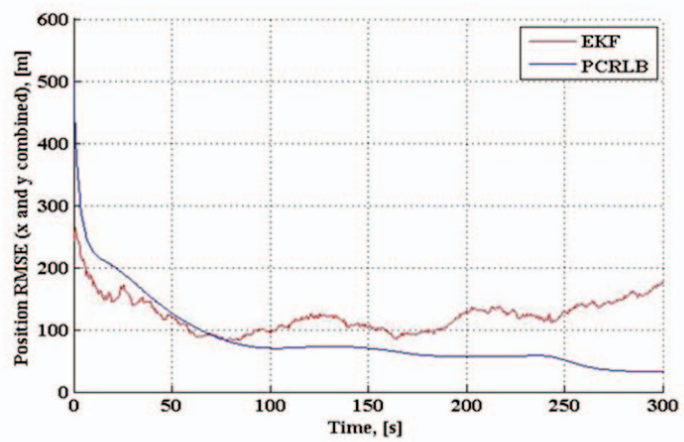

Fig. 7. Ensemble average of the position RMSE plotted with the ensemble average of the position PCRLB of the UGV node over 100 runs 


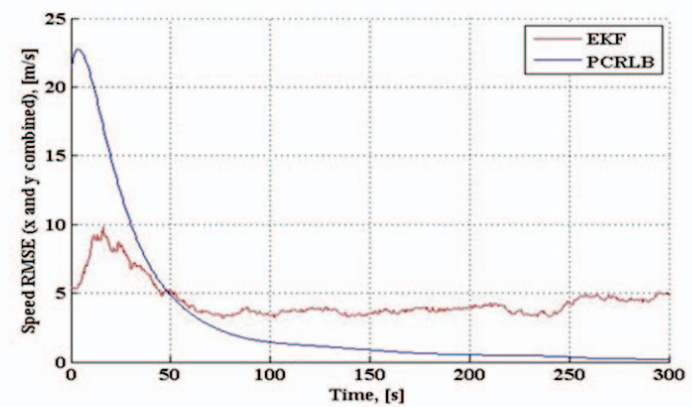

Fig. 8. Ensemble average of the velocity RMSE plotted with the ensemble average of the velocity PCRLB of the UGV node over 100 runs

The aggregate behavior of the vector of innovations was examined using the hypothesis test on the WSSR. We see from Figure 9 that the WSSR values (in blue) never exceed the WSSR threshold (in red). Because the zero-mean test, the whiteness test and the WSSR test are all satisfied, we declare that the EKF is tuned, and the overall performance is acceptable [9], [12], [13].

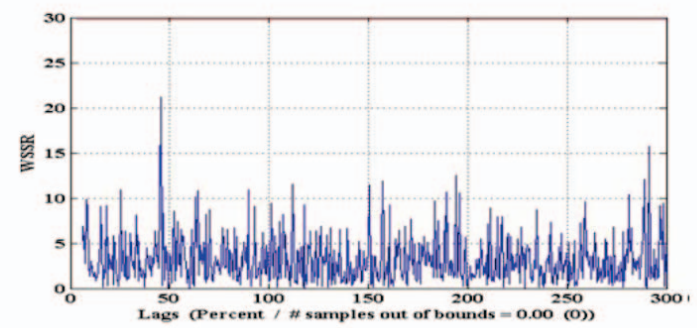

Fig. 9. The WSSR values are plotted in blue, and the WSSR threshold is plotted in red. We see that the WSSR never exceeds the threshold; so the innovations vector satisfies the WSSR hypothesis test, and we declare that the EKF is tuned

Note that the convergence speed of the EKF is highly sensitive to the choice of initial conditions. The more prior knowledge one has of the operational environment and node behavior, the better choices one can make for the initial conditions.

\section{CONCLUSION}

In this paper we have implemented a mobility estimation algorithm that can be coupled with a cooperative communication routing algorithm to provide a basis for real time path planning in UGV-DTNs. The algorithm is based on an EKF technique that exploits a non-linear Gauss Markov state model to reflect node dynamics. The EKF algorithm filters recursively, estimating the current state of the UGV node. The storage of additional past information is not required, so storage resource utilization for an individual UGV node is minimized. The algorithm is shown to implement efficient mobility tracking of UGV nodes in a wireless network and therefore can be legitimately integrated into a new cooperative routing protocol with enhanced performance. In our future work, the formulation of a path planning strategy using the mobility prediction model developed in this paper will be studied in the UGV-DTN environment.

\section{REFERENCES}

[1] R. O'Rourke, "Unmanned vehicles for u.s. naval forces: Background and issues for congress," CRS Report for Congress, October 2006, http:www.fas.org/sgp/crs/weapons/RS21294.pdf.

[2] E. Kuiper and S. Nadjm-Tehrani, "Geographical routing with location service in intermittently connected manets," IEEE Transactions on Vehicular Technology, vol. 60, no. 2, pp. 592-604, February 2011.

[3] L. Mihaylova, D. Angelova, S. Honary, D. Bull, C. Canagarajah, and B. Ristic, "Mobility tracking in cellular networks using particle filtering," IEEE Transactions on Wireless Communications, vol. 6, no. 10, pp. 3589-3599, October 2007.

[4] Z. Zaidi and B. Mark, "Mobility tracking based on autoregressive models," IEEE Transactions on Mobile Computing, vol. 10, no. 1, pp. 32-43, January 2011.

[5] J.V. Candy, Baysian Signal Processing, Classical, Modern and Particle Filtering Methods, Wiley, 2009.

[6] E. Amar and S. Boumerdassi, "A scalable mobility-adpative location service with kalman based prediction," 2011, pp. 593-598.

[7] Z. Zaidi and B. Mark, "A mobility tracking model for wireless ad hoc networks," in Proc. of IEEE WCNC, 2003, pp. 1790-1795.

[8] A. Jazwinski, Stochastic Processes and Filter Theory, Academic Press, 1970.

[9] J.V. Candy, Model-Based Signal Processing, IEEE Press and Wiley Interscience, 2006.

[10] Z. Yang and X. Wang, "Joint mobility tracking and handoff in cellular networks via sequential monte carlo filtering," IEEE Transactions on Signal Processing, vol. 51, no. 1, pp. 269-281, January 2003.

[11] R. Singer, "Estimating opticmal tracking filter performance for manned maneuvering targets," IEEE Transaction on Aerospace Electronic Systems, vol. 6, no. 4, pp. 473-483, July 1970.

[12] T.M. Beach, "Mobility modeling and estimation for delay tolerant unmanned ground vehicle networks," M.S. thesis, Naval Postgraduate School, Monterey, CA, USA, June 2013.

[13] G.A. Clark, "Angular and linear velocity estimation for a re-entry vehicle using six distributed accelerometers: Theory, simulation and feasibility," Tech. Rep., Lawrence Livermore National Laboratory UCRL-ID-153253, April 2003.

[14] B. Ristic, S. Arulampalam, and N. Gordon, Beyond the Kalman Filter: Particle Filters for Tracking Applications, Artech House, 2004. 